\title{
Are Electron Irradiation-Induced Bubbles In Amorphous Sodium Phosphate Only Transient, Or Can They Have A Long Lifetime?
}

\author{
W. H. Massover \\ Department of Biological Sciences, Rutgers University - Newark, 195 University Avenue, \\ Newark, NJ 07102
}

Radiation damage from electron beam exposure remains a fundamental problem for high resolution structure determination of both biological and materials science specimens by transmission electron microscopy (TEM) [e.g., 1,2]. Cryo-TEM and low-dose exposures for data recording are used widely to limit radiation damage for protein specimens [3]. Electron irradiation-induced bubbling in a thin amorphous layer of dried sodium phosphate buffer was reported to go through a typical early sequence of initiation, growth, fusion, and end-state [4,5]. New gas bubbles formed within solid materials are expected to have only short lifetimes with continued electron irradiation. This study examines whether the gas bubbles formed in sodium phosphate have a short or long lifetime.

A $400 \mathrm{mM}$ aqueous stock solution of sodium phosphate buffer $\left(\mathrm{NaH}_{2} \mathrm{PO}_{4} / \mathrm{Na}_{2} \mathrm{HPO}_{4}\right)$ at $\mathrm{pH} 7.0$ is freshly diluted to make a working solution of $100 \mathrm{mM}$. A small volume of $15 \mathrm{~nm}$ protein-A-coated colloidal gold particles (Ted Pella) is added to this working solution. One drop is deposited onto a 400-mesh copper grid carrying a thin carbon support film; after drainage by touching to filter paper, the grid is dried rapidly. Phase contrast imaging $(100-120 \mathrm{kV})$ by objective lens defocus uses a Tecnai-12 instrument (FEI Co.) with a cooled 4kx4k CCD camera (Gatan: Ultrascan). Specimens remain at ambient temperature $\left(20^{\circ} \mathrm{C}\right)$; no cryo-protection is utilized. Both high-dose and low-dose exposures use Tecnai software for beam blanking to prevent specimen irradiation at all other times.

Serial recordings of dynamic events in areas with moderate thickness during continued intermittent high-dose irradiation show that: (1) some initial larger bubbles remain intact during further dosage; (2) larger bubbles generated by earlier multiple fusions still contain gas (Fig. 1); (3) during the later generalized flattening, very large irregular bubbles undergo yet further fusion events, modify their shape, and often do not disappear (Fig. 1); (4) upon continued high-dose irradiation, individual smaller bubbles in the flattened areas slowly shrink and ultimately can vanish into the smooth matrix layer (Fig. 2). A thin darker boundary, probably representing condensed matrix, is imaged around all but the very smallest bubbles (Figs. 1 and 2).

The dynamic changes observed in gas bubbles within amorphous dried sodium phosphate buffer during high-dose irradiation proceed rather slowly. The two major controlling parameters for bubble evolution with further irradiation are total dose and matrix thickness (i.e., amount of substrate available for gas generation; presence and degree of vertical compression). The lighter lumen and the extensive plastic changes of size and shape now observed indicate that gas still is present inside these bubbles. These results comprise evidence that the radiolytic changes induced by high doses of transmitted electrons within amorphous dried sodium phosphate buffer produce surprisingly long-lived gas bubbles. It is proposed that the thin condensed layer bounding these bubbles acts to retard redissolution of contained gas into the surrounding matrix, or to prevent bubble escape by merging at the matrix surface facing the high vacuum inside the microscope column. Many bubbles do slowly shrink and some can disappear, but no bursting is observed. 


\section{References ---}

[1] R. F. Egerton et al., Micron. 35 (2004) 399-409.

[2] R. M. Glaeser, J. Struct. Biol. 163 (2008) 271-276.

[3] W. H. Massover, J. Synchrotron Rad. 14 (2007) 116-127.

[4] W. H. Massover, Microsc. Microanal. 12 Suppl. 2 (2006) 390CD-391CD.

[5] W. H. Massover, Proc. $16^{\text {th }}$ Internat. Cong. Microscopy (Sapporo). 1 (2006) 492.

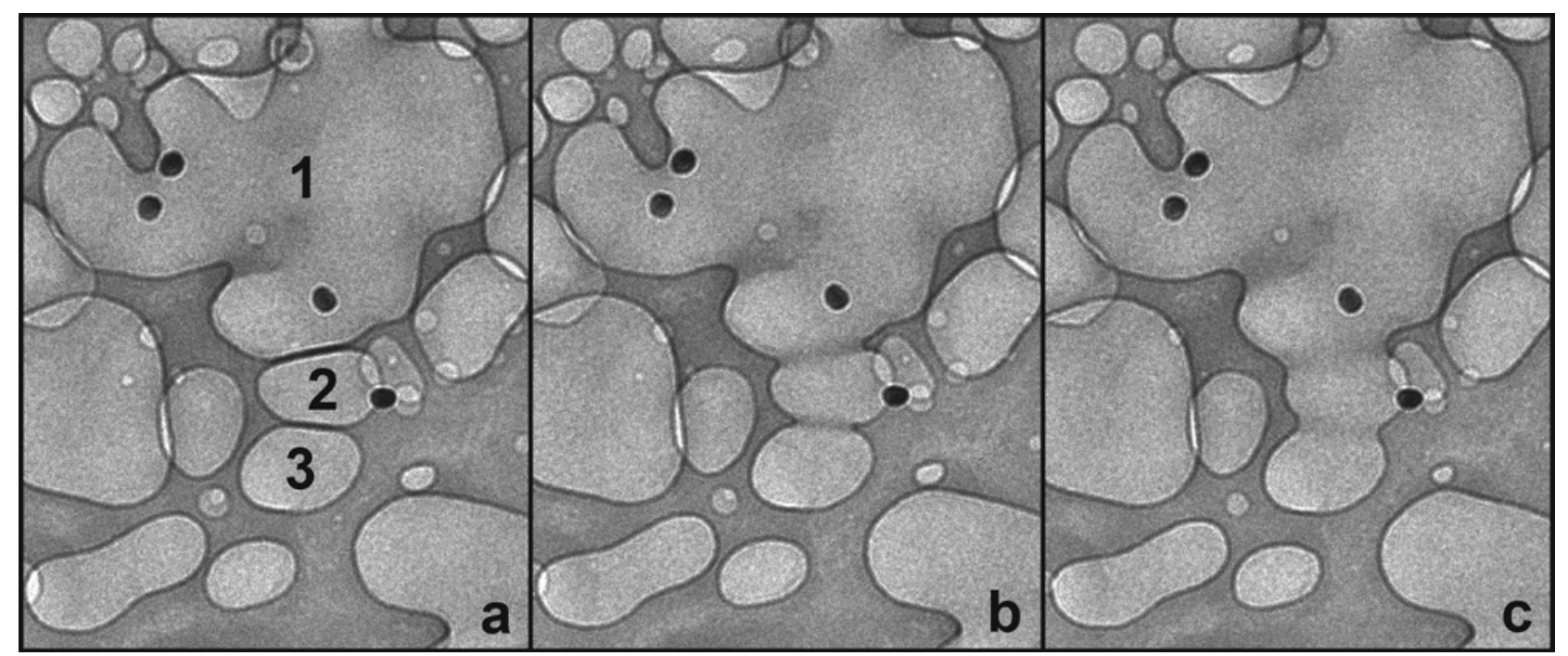

FIG. 1. Large irregular bubble (1) formed by several earlier fusions undergoes additional fusion events with two other bubbles (2 and 3). (a) High-dose exposure after general flattening occurs; same area after one (b) and two (c) identical exposures. Note smoothing of perimeter at fusion sites in $\mathrm{b}$ and $\mathrm{c}$. Four black dots are the same colloidal gold particles $(15 \mathrm{~nm})$ in all frames (a-c).

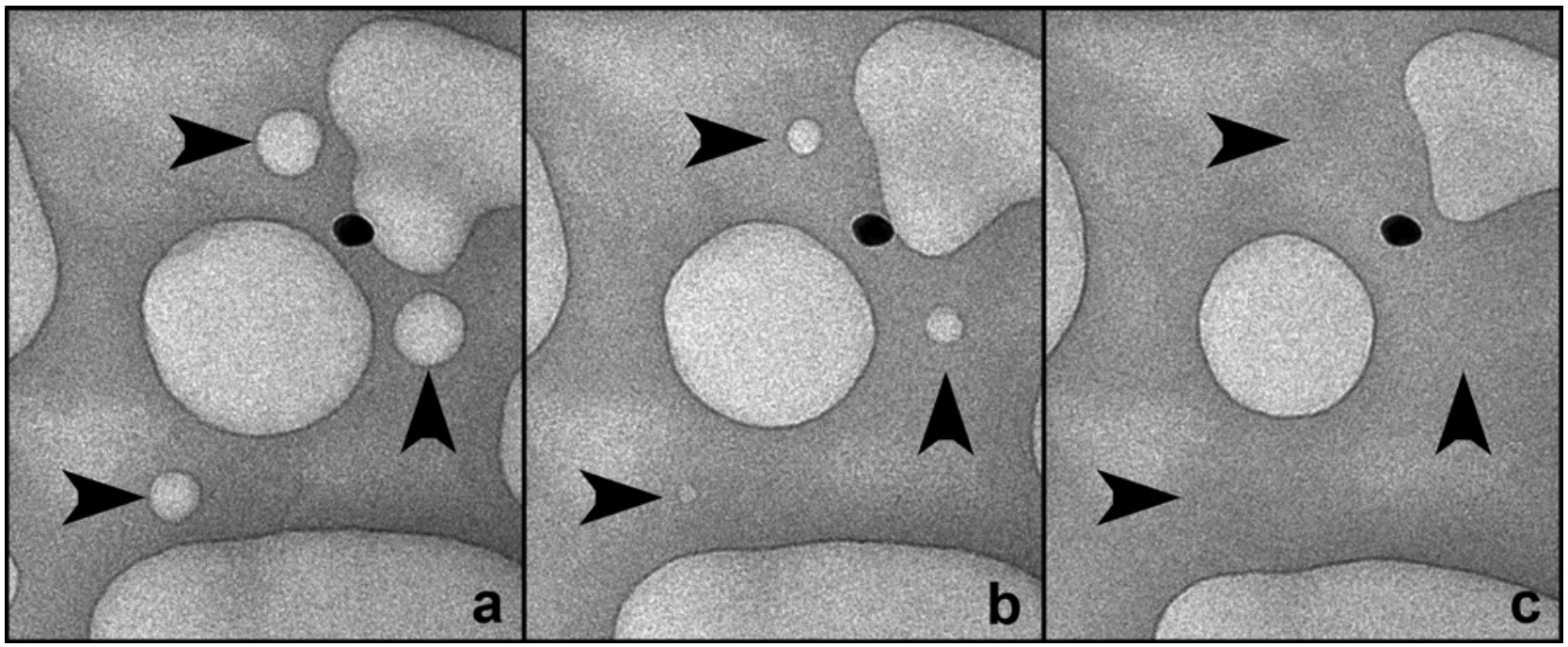

FIG. 2. Serial images showing disappearance of bubbles during continued high-dose irradiation. (a) First exposure; same area after 4 (b) and 8 (c) additional exposures. Some smaller bubbles (arrowheads) slowly shrink and vanish; adjacent larger bubbles also decrease their diameters during this additional dosage. Black dot is the same colloidal gold particle $(15 \mathrm{~nm})$ in all frames (a-c). 